\title{
Treatment of Severe Acute Bacterial Keratitis in Rabbits Using Continuous Topical Ocular Instillation with Norvancomycin
}

This article was published in the following Dove Press journal:

Drug Design, Development and Therapy

\author{
Wenxiang Lin $\mathbb{D}^{1,2}$ \\ Libei Zhao ${ }^{2}$ \\ Qian $\operatorname{Tan}^{1,2}$ \\ Ding Lin (D) ${ }^{1,2}$ \\ 'Department of Ophthalmology, \\ Changsha Aier Eye Hospital, Aier School \\ of Ophthalmology, Central South \\ University, Changsha, People's Republic \\ of China; ${ }^{2}$ Department of \\ Ophthalmology, Changsha Aier Eye \\ Hospital, Central South University, \\ Changsha, People's Republic of China
}

Purpose: Efficacy of norvancomycin (NVCM) through continuous topical ocular instillation drug delivery (CTOIDD) system for treating severe acute bacterial keratitis infection with Staphylococcus aureus was investigated.

Methods: Rabbits with bacterial keratitis were treated using CTOIDD with NVCM ( $\mathrm{n}=13)$, topical NVCM eye drops $(n=11)$, and CTOIDD with saline $(n=8)$. Clinical signs of keratitis in all groups were assessed consecutively for a week. Bacterial quantification of excised corneas was counted on the fourth and eighth days. Histopathologic examinations were performed to assess inflammatory cell infiltration on the eighth day.

Results: All signs of bacterial keratitis were alleviated in CTOIDD with NVCM according to criteria, and the CTOIDD-NVCM group had significantly less inflammation than CTOIDD-saline $(p<0.05)$, and eye drop-NVCM $(p<0.05)$. Two eyes in the eye dropNVCM group, four eyes in the CTOIDD-saline group had corneal perforation (CP), while none of the rabbits showed CP in the CTOIDD-NVCM group. Bacterial counts were significantly less in the CTOIDD with NVCM group in comparison to the eye dropNVCM $(p<0.05)$, and CTOIDD-saline $(p<0.05)$ groups. Severe inflammation and marked inflammatory cell infiltration were found in histopathologic examinations in the CTOIDDsaline and eye drop-NVCM groups, while significantly less inflammation was documented in the CTOIDD-NVCM $(p<0.05)$ group.

Conclusion: CTOIDD with NVCM effectively reduced the severity and treated acute bacterial $S$. aureus keratitis infection in a rabbit model. The presented approach of CTOIDD with NVCM appears to be a promising therapeutic approach for severe acute bacterial keratitis.

Keywords: severe acute bacterial keratitis, continuous topical instillation, eye drop, norvancomycin, rabbit eyes

\section{Introduction}

Microbial keratitis is a potentially sight-threatening emergency of corneal infection caused by bacterial, fungi, virus, or amoeba. Global estimates of microbial keratitis as a cause of monocular blindness ranges from 1.5 to 2 million new cases per year. ${ }^{1}$ In China, corneal ulceration is the most common cause of corneal blindness according to a nationwide survey. ${ }^{2}$ Bacterial keratitis is the most prevalent type with sequelae including corneal scarring, permanent visual impairment, and vision loss. While in the past bacterial keratitis infections are due to ocular trauma and poor sanitary conditions; in recent times the use of contact lenses and refractive surgeries has increased the incidence of bacteria keratitis especially methicillin-resistance Staphylococcus aureus
Correspondence: Ding Lin

Department of Ophthalmology, Changsha Aier Eye Hospital, Aier School of

Ophthalmology, Central South University,

Changsha, People's Republic of China

Tel +86-I3787255I 58

Email linding@csu.edu.cn 
keratitis. $^{3,4}$ Although some studies investigated the effectiveness of natural molecules such as essential oils (EOs) in treating multidrug resistance in pathogens, the results are primarily at the laboratory stage. ${ }^{5-9}$ Delayed treatment in severe bacterial ulcerative keratitis may result in corneal perforation, development of endophthalmitis, and loss of the eye. ${ }^{10-12}$ In addition, prompt and effective treatment of progressive keratitis requires aggressive antimicrobial therapy. $^{13}$

Topical antibiotics remain the first-line treatment for bacterial keratitis. ${ }^{14}$ Regular dose intervals of eye drop (four times per day) are not effective against severe acute infections because of the poor residue of the drug on the ocular surface due to high tear turnover, rapid nasolacrimal drainage, and limited corneal permeability. ${ }^{15,16}$ Therefore, fortified eye drops are the method of choice for treating severe cases. ${ }^{13}$ However, due to the aforementioned shortcomings, the use of topical fortified antibiotic eye drops can in instances become limited and impractical in rural areas especially in developing countries, and lead to low patient compliance and increase health-care expense as the loading dose can range from every 5 to 15 min followed by hourly instillation of topical fortified antibiotic eye drops. ${ }^{10}$

The continuous topical ocular instillation drug delivery (CTOIDD) system comprises a ring part of cribriform polyvinyl chloride tube and an attached drug pump. This device is characterized by automatic, controllable, and continuous instillation of drug solution to the ocular surface. ${ }^{17}$ Norvancomycin (NVCM) is one of the glycopeptide antimicrobial agents, has similar antibacterial characteristics to vancomycin, and is an inexpensive alternative to vancomycin. ${ }^{18-21}$

Various publications have demonstrated enhanced ocular drug delivery utilizing methods, such as liposomes, nanoparticles, contact lenses, microneedles, and an iontophoresis technique. However, their limiting factors are lower total drug dose, uncontrollable drug delivery, material biodegradation, and their adverse events have not been assessed. $^{22-27}$

The effective treatment of bacterial keratitis strongly depends on achieving adequate amounts of antibiotics to the cornea for a sufficient duration of time to control the progression of severe acute infection. In our previous pharmacokinetic study, we reported that the CTOIDD system provided higher concentrations of NVCM in nondiseased ocular tissues of rabbits compared to hourly administration of eye drops. ${ }^{17}$ Thus, this study was designed to investigate the efficacy of NVCM through CTOIDD for treating severe acute bacterial keratitis infection with $S$. aureus in rabbits.

\section{Materials and Methods}

\section{Animals}

New Zealand White female rabbits (age, 6-8 weeks; weight, $2.5-3.0 \mathrm{~kg}$ ) were obtained from the Department of Laboratory Animals, Central South University. The study conformed to the National Institutes of Health guide for the care and use of laboratory animals and was performed in accordance with the Guidelines of the Animal Ethics Committee of the Central South University (approval no. 2019sydw0045).

\section{Strains}

S. aureus (ATCC 25923) was provided by the Department of Microbiology, Central South University, Changsha, China.

\section{Bacterial Cultures}

$S$. aureus was cultured on agar plates and stored at $4{ }^{\circ} \mathrm{C}$ for use. Several colonies of this $S$. aureus strain were inoculated into $10 \mathrm{~mL}$ lysogeny broth medium and incubated at $37^{\circ} \mathrm{C}$ for $24 \mathrm{~h}$ for amplification. We used a spectrophotometer to measure the concentration and adjusted at $1 \times 10^{8}$ $\mathrm{CFU} / \mathrm{mL}$ before use.

\section{Induction of Bacterial Keratitis}

A rabbit model with bacterial keratitis was established. All the healthy rabbits with no ocular disease verified through full examination were used for the study. Each New Zealand White rabbit was anesthetized with an intramuscular injection of xylazine hydrochloride (2 mL: 0.2 g, Huamu Animal Health Products Co., Jilin, China; $0.1 \mathrm{~mL}-0.2 \mathrm{~mL} / \mathrm{kg}$ ) before surgical procedures. Left eyes were chosen as experimental eyes and topically anesthetized using proparacaine hydrochloride eye drops (15 mL: 75 mg, s.a. Alcon-Couvreur NV, Belgium), three times at three-minute intervals. Fifty microliters of $S$. aureus with a concentration of $1 \times 10^{8} \mathrm{CFU} / \mathrm{mL}$ was injected directly into the corneal stroma with a $30 \mathrm{G}$ needle in a $1 \mathrm{~mL}$ gas-tight syringe when the eye was held steady with forceps.

\section{Examination, Scoring of Rabbit Eyes, and Grouping}

Eyes were examined using a slit-lamp microscope (SL-D7, Topcon Corp., Tokyo, Japan) after 24 h of $S$. aureus injection 
under anesthesia. The eyes were observed and rated following the criteria used by Wei et al which included four clinical parameters: (1) discharge, (2) conjunctiva hyperemia/edema, (3) hypopyon and (4) cornea infiltration edema. ${ }^{28}$ Each parameter was given a grade of 0 (normal) to 4 (maximally severe), and 5, if (corneal perforation was observed). The clinical scores of bacterial keratitis grading methodology are illustrated in Table 1. The final scores for each eye were averaged after two trained blinded ophthalmologist observers graded(Dr Wenxiang Lin and Dr Libei Zhao). Rabbit eyes having the corneal score $\geq 3$ points and $<5$ points were chosen as successful keratitis model. S. aureus was again cultured from ulcers and discharges of the chosen rabbit eyes in order to verify and establish that the active infection was due to $S$. aureus. The rabbits with established keratitis model were randomly divided into three groups: CTOIDD-NVCM $(\mathrm{n}=13)$, eyedrop-NVCM $(\mathrm{n}=11)$, and CTOIDD-saline $(\mathrm{n}=8)$. All rabbits in different groups were sacrificed at different times for histopathological examination and bacteria count of corneas (Figure 1).

\section{Preparation of CTOIDD}

The CTOIDD consists of two parts, a drug delivery ring, and an automatic pump (BYZ-810T, Biyang Corp, Changsha, China). The ring part was made of a sterile polyvinyl chloride tube ring with 10 poles $(0.3 \mathrm{~mm}$ diameter) in a semicircle each. The proximate end was closed, and the distal end connected with an auto-infusion pump. ${ }^{17}$ General and topical anesthesia were the same as described in the "Induction of bacterial keratitis" subsection. The ring part was sutured to be fixed in the bulbar conjunctiva around the limbus (Figure 2).

\section{Antibacterial Regimen}

NVCM solution loaded in the CTOIDD was delivered at a rate of $2 \mathrm{~mL} / \mathrm{h}$ from the pump through the poles to the ocular surface for six hours every day in the CTOIDD-NVCM group. The six-hour delivery was kept at one time every day. The NVCM solution was observed to be distributed continuously on the ocular surface. In the eye drop-NVCM group, NVCM solution was prepared and administrated one drop every two hours, six times every day. NVCM hydrochloride solution $(50 \mathrm{mg} / \mathrm{mL})$ was freshly prepared. Norvancomycin hydrochloride (Huabei pharmaceutical Co., Ltd, China), 400 mg was mixed with $8 \mathrm{~mL}$ normal saline and used within two days. CTOIDD with normal saline loaded was applied in the CTOIDD-saline group. All rabbits were kept in individual cages with food and water during drug administration. Treatment was initiated $24 \mathrm{~h}$ after the corneal infection in the rabbits and lasted for a week. CTOIDD device would be removed after drug delivery finished every day.

\section{Evaluation of Treatment Slit-Lamp Photography}

The left eyes of each of the rabbits were observed every day using a slit lamp (SL-D7, Topcon Corp., Tokyo, Japan). Photographs were taken and two blinded observers ( $\mathrm{Dr}$ Wenxiang Lin and Dr Libei Zhao) gave scores according to the rating standard as mentioned in Table 1 on the second, fourth, and eighth days.

\section{Anterior Segment Optical Coherence Tomography (AS-OCT)}

Rabbits underwent the AS-OCT (Phoenix MICRONTM, Phoenix Technology Group, LLC, Pleasanton, CA, USA) scans to observe the perpendicular view of the cornea after treatment.

Table I Clinical Score Standard of Keratitis Infected with S. aureus

\begin{tabular}{|l|l|l|l|l|l|}
\hline Score & $\mathbf{0}$ & $\mathbf{I}$ & $\mathbf{2}$ & $\mathbf{3}$ & $\mathbf{4}$ \\
\hline Discharge & No & Small amount & Medium amount & Large amount & Upper and lower eyelid adhesion \\
\hline $\begin{array}{l}\text { Conjunctiva } \\
\text { (hyperemia/ } \\
\text { edema) }\end{array}$ & No & Slight & Mild & Moderate & Severe \\
\hline Hypopyon & No & $<$ I/4 of anterior chamber & I/4-I/2 & I/2-3/4 & $>3 / 4$ \\
\hline $\begin{array}{l}\text { Cornea } \\
\text { (infiltration/ } \\
\text { edema) }\end{array}$ & No & $\begin{array}{l}\text { Transparent cornea, slight } \\
\text { edema, scattered } \\
\text { punctuate infiltrates, } \\
\text { clearly visible iris texture }\end{array}$ & $\begin{array}{l}\text { Moderate corneal opacity, } \\
\text { infiltration area up to I/4-I/ } \\
2 \text { of the entire cornea, still } \\
\text { visible iris texture }\end{array}$ & $\begin{array}{l}\text { Severe corneal opacity, } \\
\text { corneal infiltrates up to I/ } \\
2-3 / 4 \text { of the entire cornea, } \\
\text { faint iris texture }\end{array}$ & $\begin{array}{l}\text { Infiltration area }>3 / 4 \text { of the } \\
\text { entire cornea, gray white opacity, } \\
\text { invisle iris and pupil; 5 points: } \\
\text { Corneal perforation }\end{array}$ \\
\hline
\end{tabular}




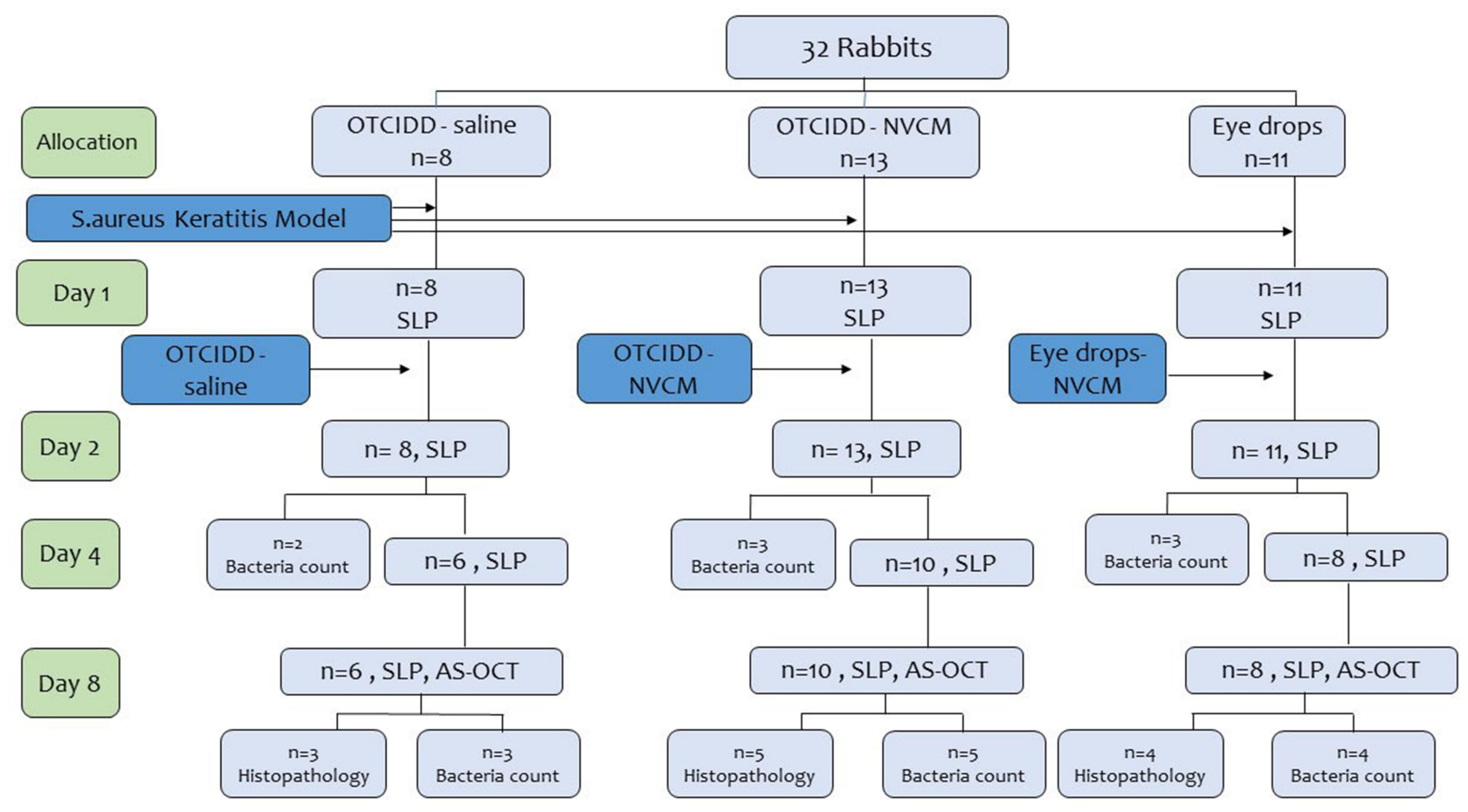

Figure I The flowchart demonstrates allocation to three groups, follow-up and investigations carried out at each time point. Abbreviations: SLP, slit-lamp photography; AS-OCT, anterior segment optic coherence tomography; CTOIDD, continuous topical ocular instillation drug delivery; NVCM, norvancomycin.

\section{Quantification of Bacterial Colony-forming Unit}

The rabbits were euthanized with an intravenous $3 \mathrm{~mL}$ lidocaine and $3 \mathrm{~mL}$ air intravenously. On the fourth and

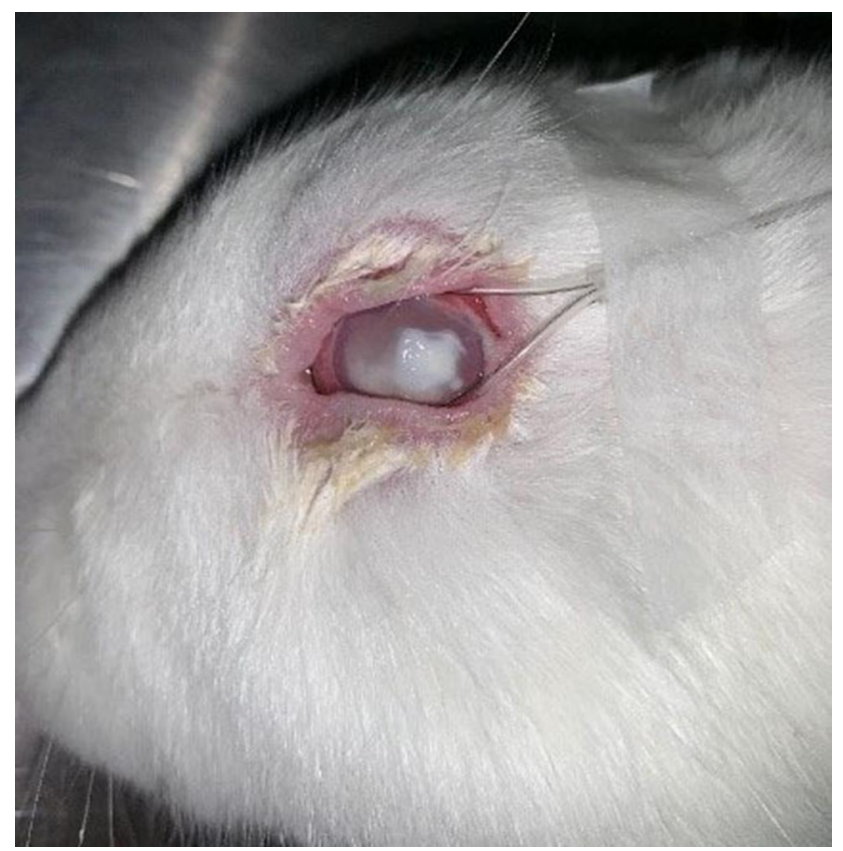

Figure 2 The ring part of the CTOIDD system model was fixed on the conjunctiva of a rabbit eye.

Abbreviation: CTOIDD, continuous topical ocular instillation drug delivery. eighth days, rabbit corneas were harvested by trephination, homogenized, and diluted serially in sterile PBS followed by fine homogenization. The homogenates were then plated in Baird-Parker plates (Comagal Technology Co., Shanghai, China). The plates were incubated at $36 \pm 1^{\circ} \mathrm{C}$ for $48 \mathrm{~h}$. The colonies were validated in the coagulase plasma test. The numbers of colonies were counted, and the results expressed as $\log 10$ number of CFU/cornea.

\section{Histopathological Evaluation}

Left eyes were enucleated after rabbits were sacrificed on the seventh day of treatment and fixed in $4 \%$ paraformaldehyde followed by dehydration with a serial concentration of ethanol (75\% for four hours, $5 \%$ for two hours, $90 \%$ for two hours, $95 \%$ for one hour, $100 \%$ for half an hour) and cleared with xylene for $35 \mathrm{~min}$. Tissues were embedded into paraffin and cut at the equatorial area with $4 \mu \mathrm{m}$ thickness using a microtome. The sections were stained with H\&E and then scanned by a Pannoramic Scanner (3DHISTECH Ltd, Budapest, Hungary). The histopathological images of ocular tissues were scored by a pathologist blinded to the experimental design. Corneal pathology scores were graded as following: a sprinkling of inflammatory cells affecting $\leq 1 / 4$ of the corneal tissue was scored as minimal (1), with dense packing of inflammatory cells affecting $1 / 4-1 / 2,1 / 2-3 / 4$, and $\geq 3 / 4$ of 
corneal stromal tissue was recorded as mild, moderate and severe $(2,3,4)$ respectively. ${ }^{29,30}$ The iris and ciliary body were graded for signs of swelling and inflammatory cell infiltration from normal (0) to severe (4).

\section{Statistical Analysis}

Statistical analyses were performed by SPSS 24.0 (IBM Corp., Armonk, NY, USA). Clinical inflammation score and pathological score were presented as median (IQR) and bacterial loads among the CTOIDD group were presented as mean \pm SD. The clinical inflammation score and pathological scores were analyzed using Kruskal-Wallis test. Dunn's test was used to compare the differences between groups. Bacterial counts were analyzed using a one-way analysis of variance. The value $p<0.05$ was considered a significant difference.

\section{Results}

Slit-lamp Photography and Clinical Scores of Eyes

All left eyes presented a marked response with acute severe keratitis including purulent secretion in the cul-de-sac, conjunctival hyperemia, and edema, deep corneal infiltration, and hypopyon within $24 \mathrm{~h}$ after the induction. Slit-lamp photography of experimental eyes was performed on days one, two, four, and eight. Figure 3 shows representative pictures of eyes in three groups before and after corresponding treatment. The eye in the CTOIDD-NVCM group presents more decrease of the hypopyon, alleviated edema of cornea, and reduction of corneal infiltration (Figure 3A-D) in comparison to the eye drop group (Figure $3 \mathrm{E}-\mathrm{H}$ ). The eye in CTOIDD-saline group displays an ulcer, deteriorating with increased inflammation and a typical perforation on day four
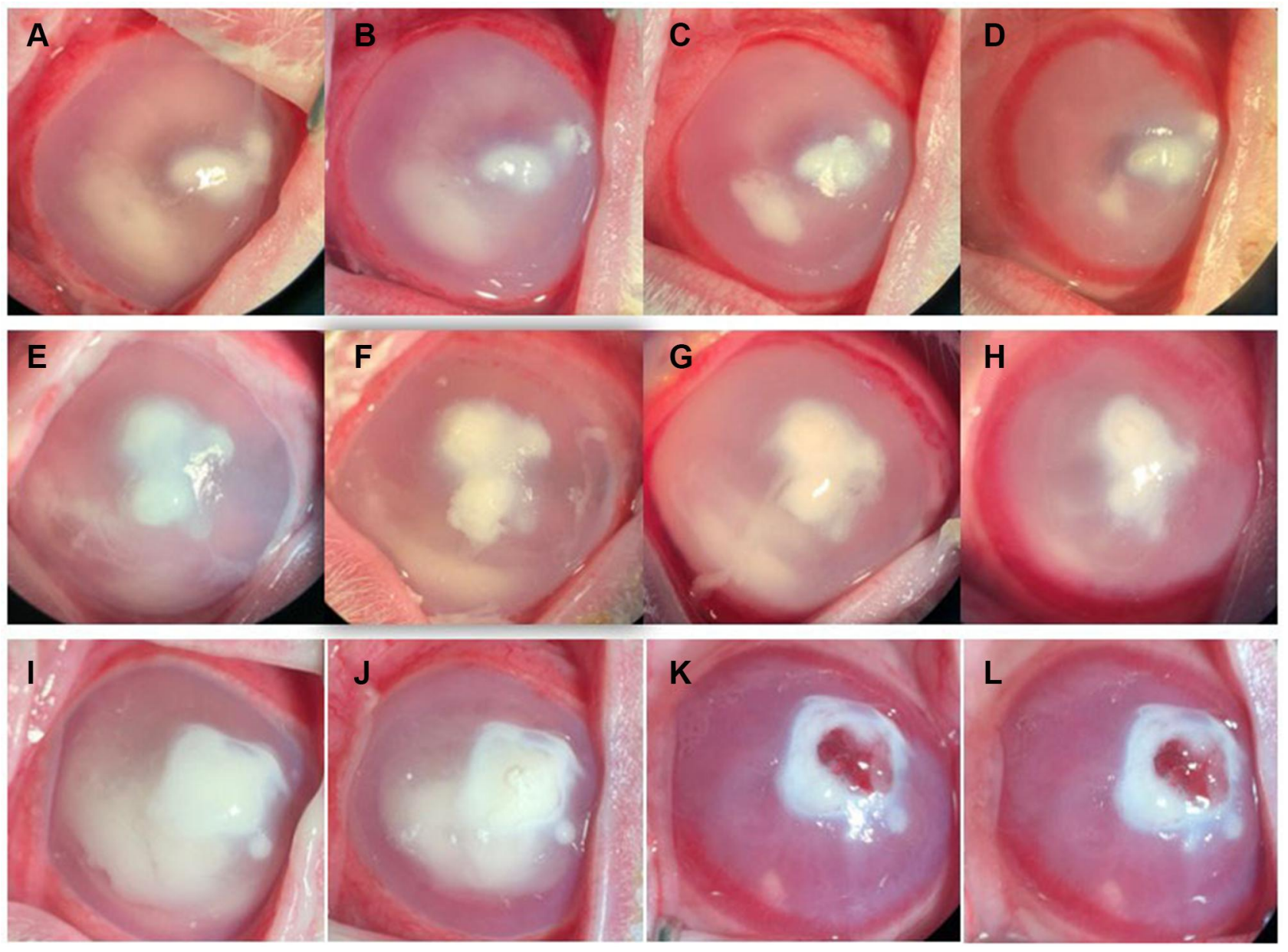

Figure 3 Representative slit-lamp pictures of the CTOIDD-NVCM group (A-D), Eye drop group (E-H), CTOIDD-saline group (I-L). Pictures were taken on days one (A, $\mathbf{E}, \mathbf{I})$, two $(\mathbf{B}, \mathbf{F}, \mathbf{J})$, four $(\mathbf{C}, \mathbf{G})$ and eight $(\mathbf{D}, \mathbf{H}, \mathbf{L})$. Eyes in the CTOIDD-saline group presented a remarkable corneal edema and infiltration, conjunctival hyperemia and hypopyon. A typical corneal perforation with hyperemic iris incarceration on day six was observed in the CTOIDD-saline group (K). The eyes on day eight in the CTOIDDNVCM and eye drop groups appeared less inflamed. 


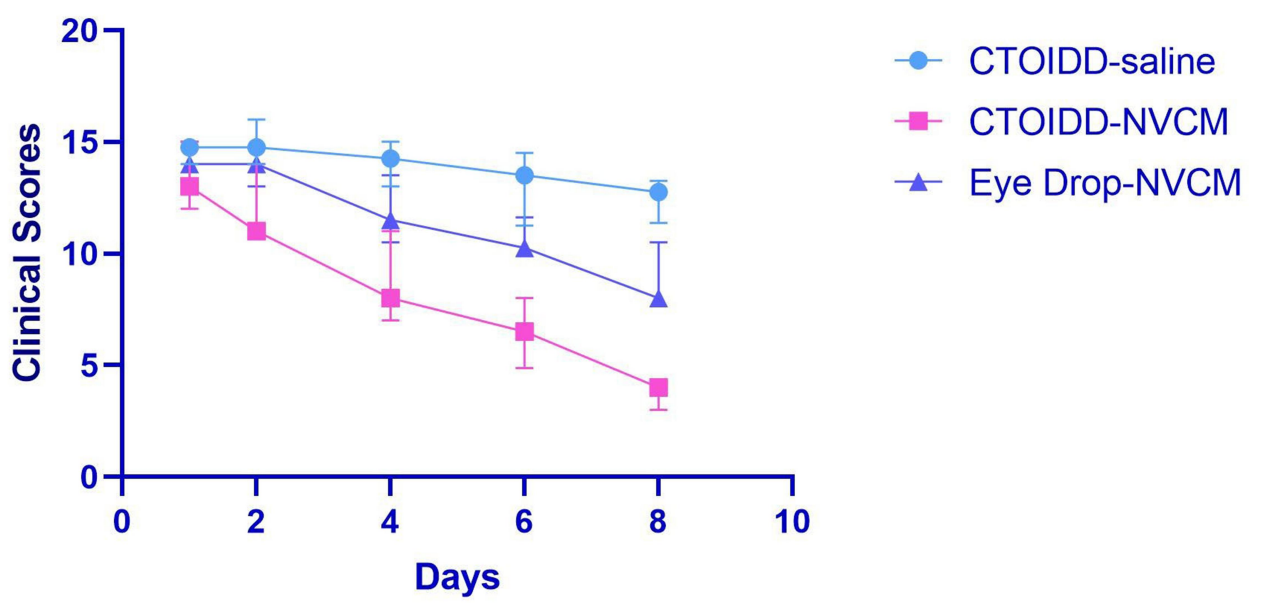

Figure 4 Clinical inflammation score changes in three groups $(n=13$ in CTOIDD-NVCM, $n=11$ in eye drop-NVCM, $n=8$ in CTOIDD-saline group on days one, two; $n=10$ in CTOIDD-NVCM group, $\mathrm{n}=8$ in eye drop-NVCM group, $\mathrm{n}=6$ in CTOIDD-saline group on days four, six, eight). Values are given as the median (IQR).

(Figure 3I-K). Figure 4 shows the total clinical scores of changes for all clinical parameters in three groups. And the data on scores of individual parameters are shown as median (IQR) in Table 2. The clinical scores before treatment among the three groups have no significant differences on day one ( $p=0.395$ ). The clinical scores of the CTOIDD-NVCM group

Table 2 Clinical Scores in Three Groups

\begin{tabular}{|l|l|l|l|l|}
\hline Parameters & Days & $\begin{array}{l}\text { CTOIDD- } \\
\text { Saline }\end{array}$ & $\begin{array}{l}\text { CTOIDD- } \\
\text { NVCM }\end{array}$ & $\begin{array}{l}\text { Eye Drop- } \\
\text { NVCM }\end{array}$ \\
\hline Discharge & 0 & $4(3.6,4)$ & $4(4,4)$ & $4(3,4)$ \\
\hline Conjunctiva & 0 & $4(3.6,4)$ & $4(3,4)$ & $3(3,4)$ \\
\hline Cornea & 0 & $4(3.6,4)$ & $3(3,4)$ & $4(4,4)$ \\
\hline Hypopyon & 0 & $3(3,3)$ & $3(1.5,3.5)$ & $3(3,4)$ \\
\hline Total & 0 & $14.75(14,15)$ & $13(12,15)$ & $14(13,15)$ \\
\hline Discharge & 4 & $3(3,3.75)$ & $2(1.75,2)$ & $2.5(2,3)$ \\
\hline Conjunctiva & 4 & $3(3,3.75)$ & $2(2,3)$ & $2.5(2,3)$ \\
\hline Cornea & 4 & $4(3.63,4.75)$ & $3(2,3)$ & $4(3,4)$ \\
\hline Hypopyon & 4 & $4(3.25,4)$ & $2(1,3)$ & $3(3,4)$ \\
\hline Total & 4 & $14.25(13,15)$ & $8(7,11)$ & $11.5(10.5,13.5)$ \\
\hline Discharge & 8 & $2.5(2,3)$ & $0(0,0)$ & $1(1,1.75)$ \\
\hline Conjunctiva & 8 & $2.5(2,3)$ & $1(\mathrm{I}, 1)$ & $2(2,2)$ \\
\hline Cornea & 8 & $5(3.38,5)$ & $2(2,2)$ & $3(3,4.75)$ \\
\hline Hypopyon & 8 & $3(3,3)$ & $1(0.88,1)$ & $2(2,2)$ \\
\hline Total & 8 & $12.75(11.38$, & $4(3,4.25)$ & $8(8,10.5)$ \\
\hline
\end{tabular}

decreased more than the CTOIDD-saline group with a significant difference on day two $(p=0.000)$, day four $(p=0.000)$, and day eight $(p=0.000)$, respectively. When compared to the eye drop-NVCM group, scores of CTOIDD-NVCM show no significant differences on day two $(p=0.095)$ and day four $(p=0.053)$, however, the scores dropped down on day eight $(p=0.018)$ with significant differences (Figure 5).

\section{Anterior Segment Optical Coherence Tomography}

Experimental eyes in three groups underwent the ASOCT examination on days one and eight, respectively. Figure 6A shows the normal structure of the cornea in a healthy rabbit. The five layers of the cornea including epithelium, Bowman's membrane, stroma, Descemet's membrane, and endothelium are distinct and intact. The epithelium and stroma in the eye drop-NVCM group are more irregular than the CTOIDD-NVCM group, besides, the endothelium in the eye drop group cannot be clearly observed (Figure 6B and C). An eye in the CTOIDDsaline group shows a conspicuous perforation in the central cornea, following corneal edema, discontinued epithelium and stroma, ruptured endothelium and fibrin in the anterior chamber (Figure 6D).

\section{Bacterial Burden}

$\log 10$ mean bacterial counts are illustrated in Figure 7. The $\log 10$ mean counts in CTOIDD-NVCM show a significant reduction compared to the eye drop group $(p=0.013$ on day 


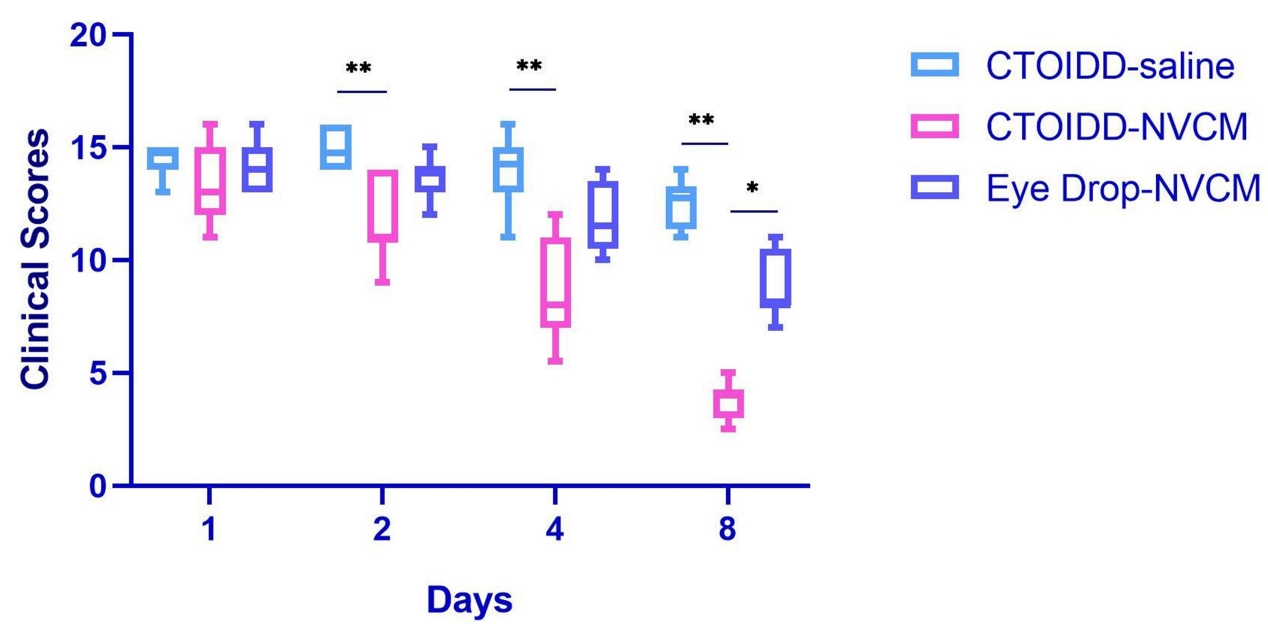

Figure 5 Comparison of inflammation scores in different days among the three groups. Kruskal-Wallis test was used to compare the difference with a posthoc test. Values are given as the median (IQR), ${ }^{*} p<0.05,{ }^{*} p<0.01$.

four; $p=0.000$ on day eight) and the CTOIDD-saline group ( $p=0.003$ on day four; $p=0.000$ on day eight). There is no significant difference between the eye drop and CTOIDDsaline groups on day four $(p=0.103)$, while bacterial counts show lower in the eye drop group than CTOIDD-saline group on day eight $(p=0.000)$.

\section{Histopathology}

Histopathological images of cornea, iris, and ciliary body in three groups on day eight are presented in Figure 8. The stroma of the cornea was thickened in all groups compared to the healthy cornea. Figure $8 \mathrm{D}$ shows a typical corneal perforation in the CTOIDD-saline group. In the CTOIDDNVCM group, the epithelium was less irregular, and the stroma was infiltrated by less polymorphonuclear neutrophil (PMN) cells than the eye drop group (Figure 8B and C). Figure $8 \mathrm{E}$ presents a worse corneal ulcer close to the endothelial layer in the eye drop group. Examination of the iris and ciliary body revealed numerous PMN cells in the CTOIDD-saline group (Figure 8I and M). However, the PMN cells appeared to be fewer in the CTOIDD-NVCM group when compared to the eye drop group.

The pathology score comparison of cornea, iris, and ciliary body in the three groups are shown in Figure 9. The scores (median, IQR) of cornea, iris, and ciliary body in the three groups are shown in Table 3. Cornea, iris, and ciliary body scores in the CTOIDD-NVCM group were significantly lower than the CTOIDD-saline group (cornea: $p=0.02$; iris: $p=0.034$; ciliary body: $p=0.037)$. There are no differences between the CTOIDD-NVCM and eye drop-NVCM groups ( $p>0.05)$.

\section{Discussion}

The findings from this study demonstrate that continuous topical ocular instillation of NVCM using CTOIDD inhibited the development of severe acute bacterial keratitis. Drug delivery of NVCM using CTOIDD was able to treat severe acute bacterial keratitis infected by $S$. aureus effectively with significantly decreasing clinical scores, reduced bacterial burden and lessened inflammatory cell infiltration in ocular tissues in a week compared to the eye drop and CTOIDD-saline groups.

At baseline, the bacterial keratitis models in our study presented with more severe symptoms of acute keratitis in comparison to other keratitis models such as topical corneal scratch and inoculation methods. ${ }^{31}$ Severe symptoms cannot be maintained for an extended period with topical corneal scratch methodology since fewer bacteria adhere to the cornea, wash-out effect by tear, and antibacteria activity by lysozyme in the tear film. Since the severity of infection is related to the concentration and total amount of bacteria, ${ }^{28,32,33}$ injecting $50 \mu \mathrm{L}$ S. aureus with a concentration of $1 \times 10^{8} \mathrm{CFU} / \mathrm{mL}$ directly into the corneal stroma enabled our models to induce severe acute bacterial keratitis. Early infection symptoms such as a small amount of discharge, mild corneal hyperemia, and infiltration in cornea occurred in six hours after inoculation of $S$. aureus followed by a marked corneal infiltration and hypopyon within $24 \mathrm{~h}$ after inoculation.

In our previous pharmacokinetic study, we used a CTOIDD system to deliver NVCM into the ocular surface to achieve a much higher drug concentration in the ocular tissue rapidly compared to hourly administration of eye 

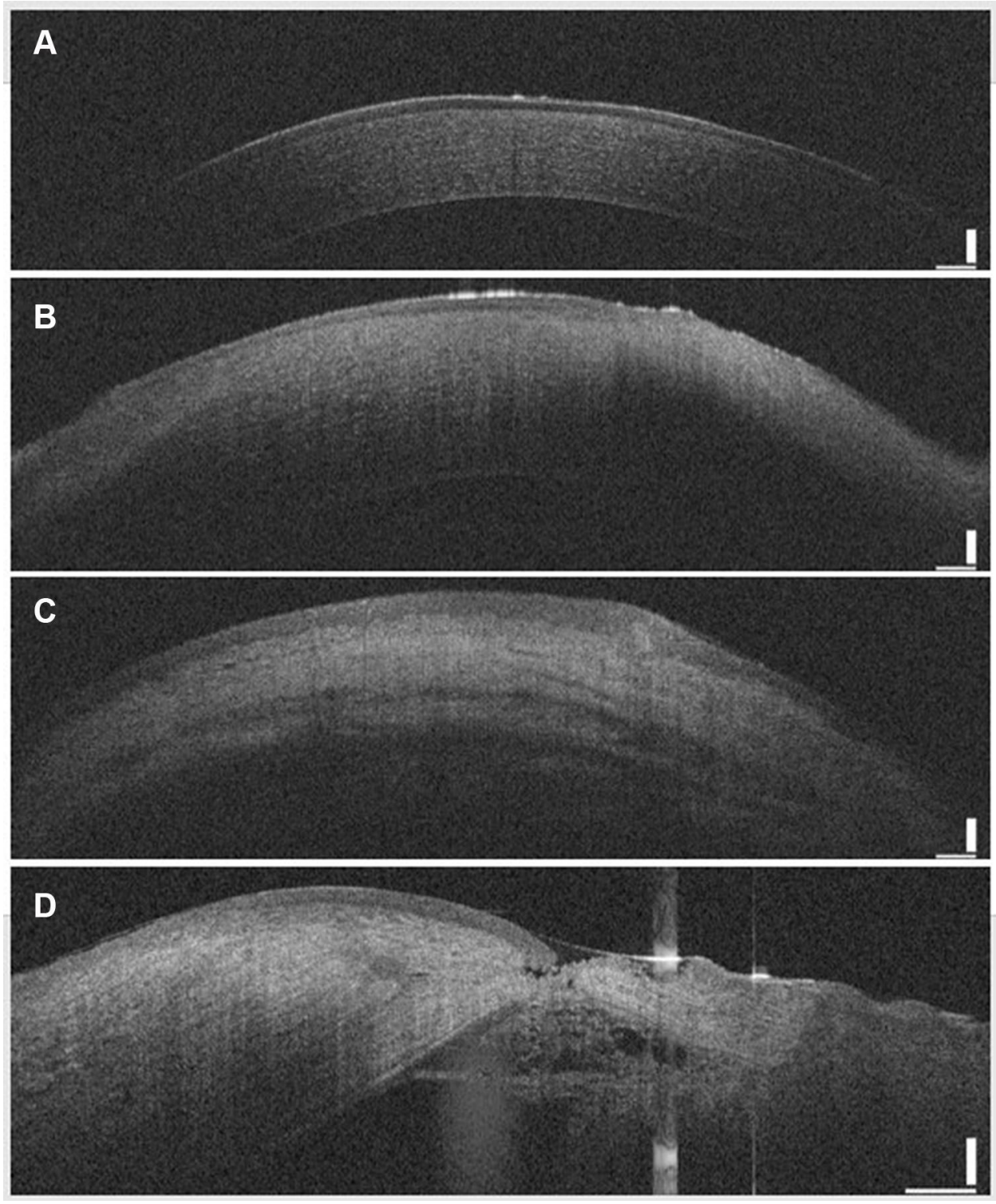

Figure 6 Representative pictures of AS-OCT examination in cornea. Cornea in healthy rabbit (A). Corneas in CTOIDD-NVCM group (B), eye drop group (C) and CTOIDD-saline group (D) on day eight, respectively.

drops in healthy rabbits. ${ }^{17}$ In this current study, we explored the efficacy of CTOIDD of NVCM for treating severe acute bacterial keratitis infection with S. aureus. Rabbits with severe acute bacterial keratitis were treated with continuous instillation of NVCM solution $(50 \mathrm{mg} /$ $\mathrm{mL}$ ) through CTOIDD for six hours per day in a one-week regimen. Rating standards used by Wei et $\mathrm{al}^{28}$ were referred for the clinical scores. The total clinical scores were the sum of these four scores including discharge, conjunctiva, hypopyon, and cornea parameters. The total clinical scores in the CTOIDD-NVCM group decreased significantly over time from day two onwards when compared with the CTOIDD-saline and eye drop-NVCM groups. Especially the cornea scores in the eye drop and CTOIDD-saline groups were significantly higher than the CTOIDD-NVCM group.

The eyes of rabbits in the CTOIDD-NVCM group presented the mildest inflammation while two eyes in the eye drop group and four in the CTOIDD-saline group developed corneal perforation according to the slit-lamp examination and AS-OCT. Besides, corneas in the CTOIDD-NVCM group carried the lightest bacterial burden on days four and eight in the quantitative assessment. The histopathological examination resulted in the CTOIDD-NVCM group presented anterior ocular tissues including cornea, iris, ciliary body had the least 


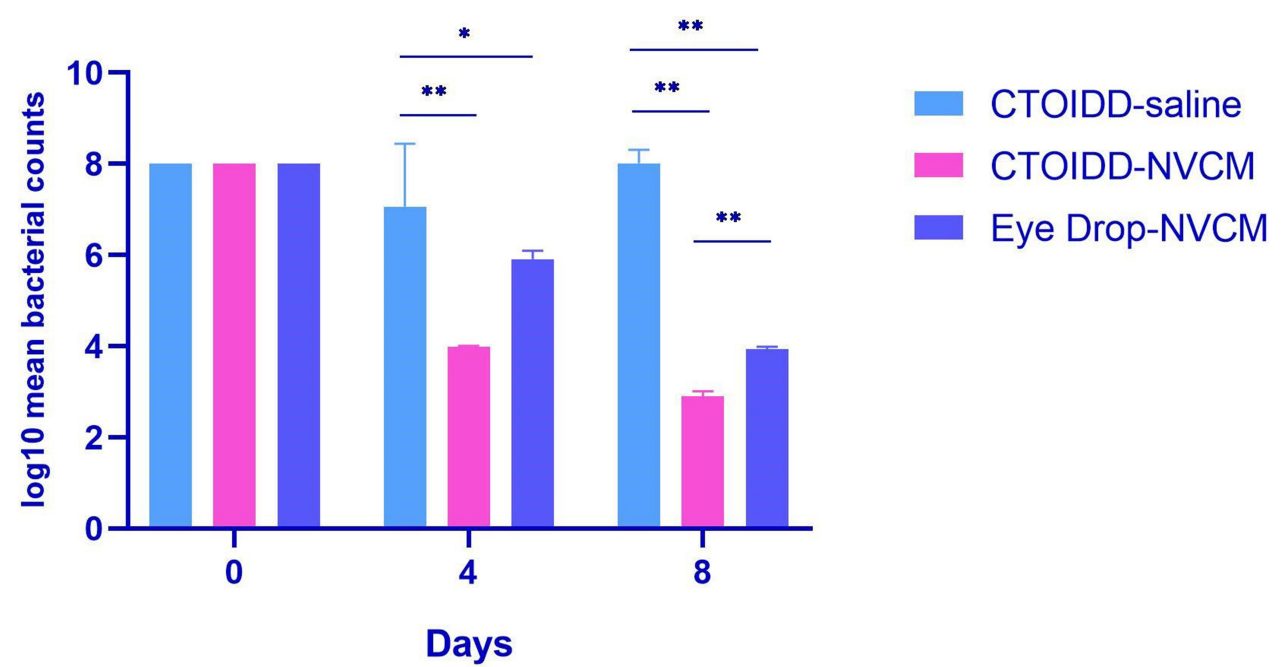

Figure 7 Comparison of bacteria count in the three groups. One-way analysis of variance was used to compare the difference with a post hoc test. Values are given as mean \pm SD, $* p<0.05, * * p<0.01$.

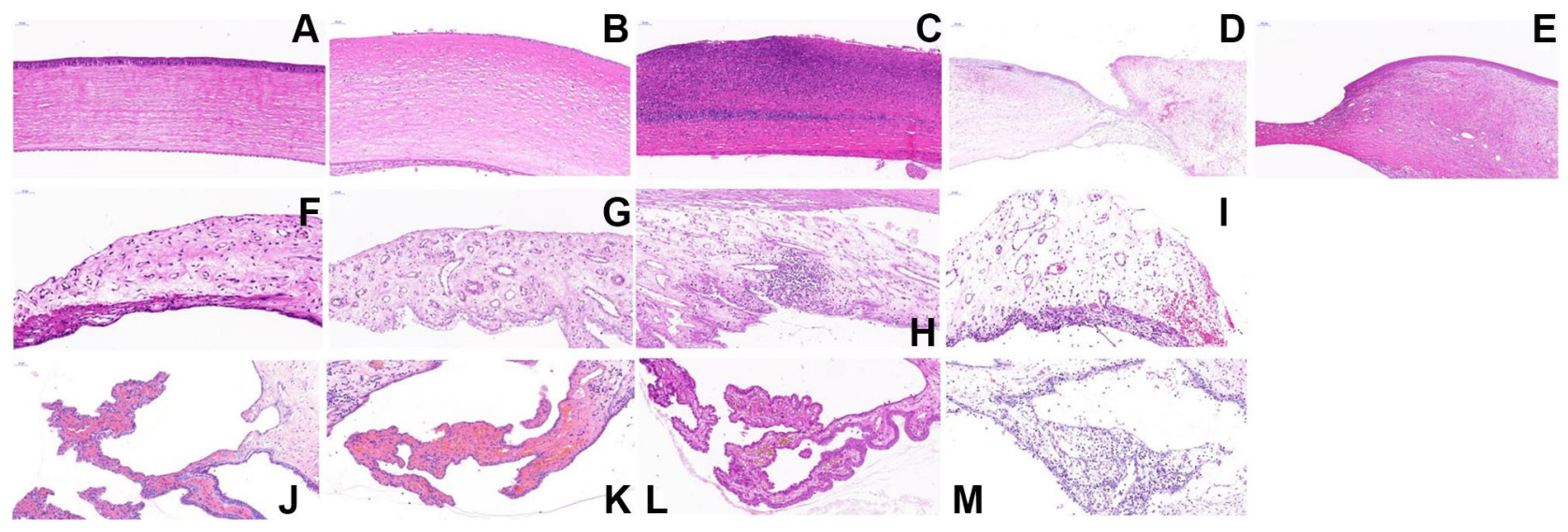

Figure 8 Representative pictures of histopathological section images of cornea (A-E), iris $(\mathbf{F}-\mathbf{I})$ and ciliary body (J-M) in healthy rabbits and three groups on day eight. Healthy cornea (A), cornea in CTOIDD-NVCM group (B), eye drop group (C, E), CTOIDD-saline group (D). Healthy iris (F), iris in CTOIDD-NVCM group (G), eye drop group (H), CTOIDD-saline group (I). Healthy ciliary body (J), ciliary body in CTOIDD-NVCM group (K), eye drop group (L), CTOIDD-saline group (M). (A-C) 20x magnification, scale bar $50 \mu \mathrm{m}$; (D, E) $10 \times$ magnification, scale bar $100 \mu \mathrm{m}$; (F-M) 20x magnification, scale bar $50 \mu \mathrm{m}$, H\&E stain.

inflammatory cell infiltration and corneal structural disruption. We also observed that frequent NVCM eye drops had benefits compared to the saline-control group, however, it may not be enough for the urgent cases in severe acute keratitis.

Recently, new strategies in topical ocular drug delivery systems have been improved to deal with poor compliances due inconvenience and difficulty in self-administering eye drops by patients and less efficacy in severe cases. A case report ${ }^{34}$ introduced the use of Morgan Lens in the treatment of Pseudomonas keratitis which was used as an irrigation device for chemical exposure before. The infusion of ceftazidime by Morgan Lens effectively treated two patients with severe keratitis. However, direct infusion of ceftazidime can irritate patients since the drug comes into contact with the cornea rapidly and directly during continuous lavage. Kesavan et $\mathrm{al}^{35}$ investigated an ionactivated mucoadhesive hydrogel system for treating experimental bacterial keratitis. It showed significant improvement in the observed parameters of bacterial keratitis in the mucoadhesive system compared to the conventional eye drop of gatifloxacin. Similar to our study, they also used S. aureus (ATCC 25923) for inducing bacterial keratitis. However, the amount of bacteria used by Kesavan et al was less (1000 CFU) in comparison to our study $\left(5 \times 10^{6} \mathrm{CFU}\right)$, since we wanted to build an acute severe keratitis model. Thus, for now, the mucoadhesive system treated in severe cases has not been investigated. 


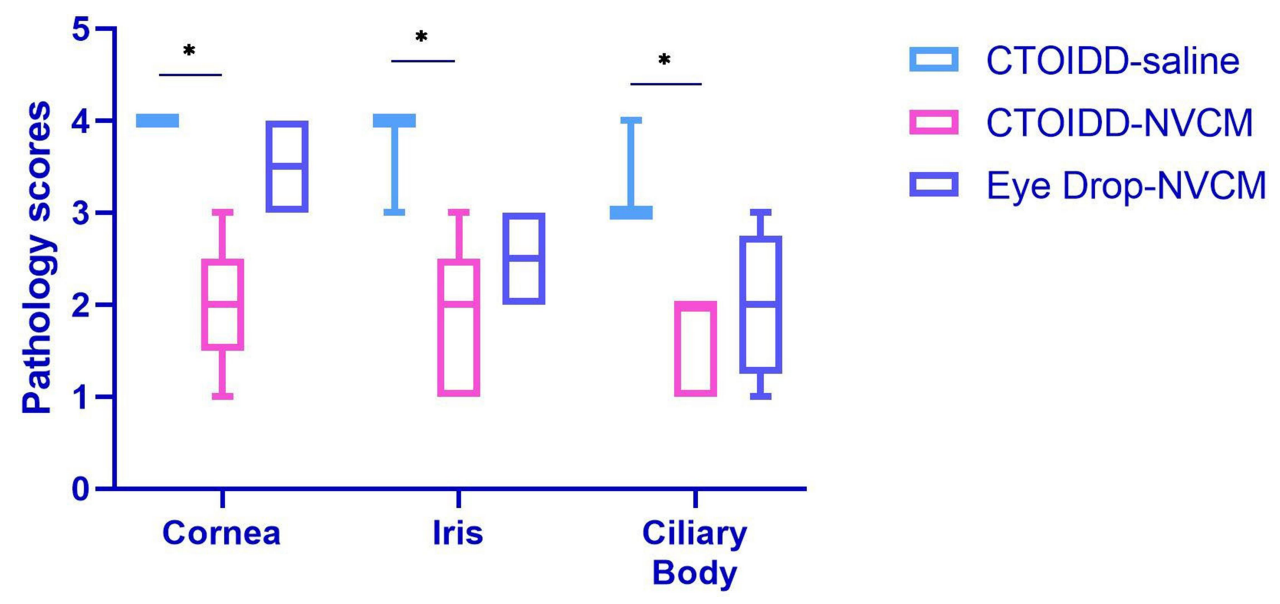

Figure 9 Comparison of pathology infiltration inflammation scores in three groups. Kruskal-Wallis test was used to compare the difference with a post hoc test. Values are given as the median (IQR), ${ }^{*} p<0.05$.

Although other various new drug delivery systems including hydrogels, nanoparticles, and microemulsions ${ }^{13,33,36}$ have demonstrated possible utility in treating mild and chronic cases, but few have shown their effectiveness against severe acute bacterial keratitis. Pinna et $\mathrm{al}^{37,38}$ conducted two studies to assess in vitro antimicrobial activity of a new commercial ophthalmic solution containing povidone-iodine $0.6 \%$ (IODIM $^{\circledR}$ ) and another containing hexamidine diisethionate $0.05 \%$ (Keratosept) and the results suggested that they may be potential candidates for the treatment of ocular surface infections. In addition, plant-based products such as EOs have also been explored for their antimicrobial properties ${ }^{5,7}$ and the beneficial properties of EOs have encouraged researchers to use them along with nanomaterials as potential antimicrobial agents. However, the promising antimicrobial activity of EOs, for now, is limited due to their strong organoleptic flavor, low water solubility, and low stability. ${ }^{39}$

The CTOIDD method provided a possible way to form a "drug film" that prolongs pre-corneal and pre-conjunctival residue times of the drug to diminish the effects of high turnover rates of the tear film. In addition, the absence of in

Table 3 Pathological Scores of Cornea, Iris and Ciliary Body in Three Groups

\begin{tabular}{|l|l|l|l|}
\hline Tissue & $\begin{array}{l}\text { CTOIDD- } \\
\text { Saline }\end{array}$ & $\begin{array}{l}\text { CTOIDD- } \\
\text { NVCM }\end{array}$ & $\begin{array}{l}\text { Eye Drop- } \\
\text { NVCM }\end{array}$ \\
\hline $\begin{array}{l}\text { Cornea } \\
\text { Iris }\end{array}$ & $4(4,4)$ & $2(1.5,2.5)$ & $3.5(3,4)$ \\
$\begin{array}{l}\text { Ciliary } \\
\text { body }\end{array}$ & $3(3,4)$ & $2(1,2.5)$ & $2.5(2,3)$ \\
\hline
\end{tabular}

vivo adverse events in the CTOIDD group confirms its safety. The results also demonstrated that continuous instillation of large drug doses can effectively control the development in the early stage of severe keratitis and provides better effectiveness than a frequent eye drop regimen.

Nonetheless, there are various limitations in our study. We admit some characteristic differences exist between the human and rabbit eyes. Rabbits have a larger and thinner cornea, more space in the cul-de-sac, faster blood circulation than humans. Thus, the animal model of experimental keratitis would differ from keratitis in humans slightly. Furthermore, the suture in our study was used to fix the ring part of the CTOIDD to keep it from dropping out because of the rabbits' proptosis. And we did not limit rabbits' activities in this study so the results could have been influenced. The CTOIDD device is still investigational. Future modifications to the CTOIDD system would allow a device suitable for the human eye when administered to patients. It would be noninvasive and conveniently placed in the cul-de-sac and the drug delivery process would be automated. Studies will investigate the safety and efficacy of CTOIDD viral and fungal disease models in the future.

\section{Conclusion}

The present study provides promising results for continuous topical instillation delivery of NVCM in preventing the progression in the early stage of severe acute bacterial keratitis in a rabbit model. The CTOIDD system demonstrates improved efficacy and has the possibility to increase patient compliance. Therefore, CTOIDD is a possible alternative approach for the treatment of severe acute corneal infections. 


\section{Acknowledgments}

We would like to thank Dr Eric E Pazo for the linguistic assistance. We would like also to thank Aier School of Ophthalmology of Central South University and the Department of Laboratory Animals of Central South University for their great help and support. This research was funded by the Key program for Hunan Provincial Science and Technology Department (No. Kc1701045). The abstract of this paper was presented at the ARVO annual meeting as a poster presentation with interim findings. The poster's abstract was published in Investigative Ophthalmology \& Visual Science. https://iovs.arvojour nals.org/article.aspx?articleid=2767962.

\section{Disclosure}

The authors report no conflicts of interest in this work.

\section{References}

1. Ung L, Bispo PJM, Shanbhag SS, et al. The persistent dilemma of microbial keratitis: global burden, diagnosis, and antimicrobial resistance. Surv Ophthalmol. 2019;64:255-271. doi:10.1016/j. survophthal.2018.12.003

2. Song X, Xie L, Tan X, et al. A multi-center, cross-sectional study on the burden of infectious keratitis in China. PLoS One. 2014:9. doi:10.1371/journal.pone. 0113843 .

3. Asbell PA, Sanfilippo CM, Pillar CM, et al. Antibiotic resistance among ocular pathogens in the united states. JAMA Ophthalmol. 2015;133:1445. doi:10.1001/jamaophthalmol.2015.3888

4. Mah FS, Davidson R, Holland EJ, et al. Current knowledge about and recommendations for ocular methicillin-resistant Staphylococcus aureus. J Cataract Refract Surg. 2014;40:1894-1908. doi:10.1016/j. jcrs.2014.09.023

5. Donadu M, Usai D, Pinna A, et al. In vitro activity of hybrid lavender essential oils against multidrug resistant strains of pseudomonas aeruginosa. J Infect Dev Ctries. 2018;12:9-14. doi:10.3855/jidc.9920

6. Donadu MG, Le NT, Ho DV, et al. Phytochemical compositions and biological activities of essential oils from the leaves, rhizomes and whole plant of hornstedtia bella Skorničk. Antibiotics. 2020;9:1-16. doi:10.3390/antibiotics9060334

7. Cannas S, Usai D, Pinna A, et al. Essential oils in ocular pathology: an experimental study. $J$ Infect Dev Ctries. 2015;9:650-654. doi: $10.3855 /$ jidc. 6842

8. Le NT, Donadu MG, Ho DV, et al. Biological activities of essential oil extracted from leaves of Atalantia sessiflora Guillauminin Vietnam. J Infect Dev Ctries. 2020;14:1054-1064. doi:10.3855/ JIDC. 12469

9. Chaves-López C, Usai D, Donadu MG, et al. Potential of: borojoa patinoi Cuatrecasas water extract to inhibit nosocomial antibiotic resistant bacteria and cancer cell proliferation in vitro. Food Funct. 2018;9:2725-2734. doi:10.1039/c7fo01542a

10. Lin A, Rhee MK, Akpek EK, et al. Bacterial keratitis preferred practice pattern ${ }^{\circledR}$. Ophthalmology. 2019;126:P1-P55. doi:10.1016/j. ophtha.2018.10.018

11. Henry CR, Flynn HW, Miller D, et al. Infectious keratitis progressing to endophthalmitis: a 15-year study of microbiology, associated factors, and clinical outcomes. Ophthalmology. 2012;119(12):24432449. doi:10.1016/j.ophtha.2012.06.030
12. Jin H, Parker WT, Law NW, et al. Evolving risk factors and antibiotic sensitivity patterns for microbial keratitis at a large county hospital. Br J Ophthalmol. 2017;101(11):1483-1487. doi:10.1136/bjophthalmol-2016-310026

13. Sharma A, Taniguchi J. Review: emerging strategies for antimicrobial drug delivery to the ocular surface: implications for infectious keratitis. Ocul Surf. 2017;15(4):670-679. doi:10.1016/j. jtos.2017.06.001

14. Austin A, Lietman T, Rose-Nussbaumer J. Update on the Management of Infectious Keratitis. Ophthalmology. 2017;124 (11):1678-1689. doi:10.1016/j.ophtha.2017.05.012

15. Awwad S, Mohamed Ahmed AHA, Sharma G, et al. Principles of pharmacology in the eye. Br J Pharmacol. 2017;174:4205-4223. doi:10.1111/bph.14024

16. Agrahari V, Mandal A, Agrahari V, et al. A comprehensive insight on ocular pharmacokinetics. Drug Deliv Transl Res. 2016;6(6):735-754. doi:10.1007/s13346-016-0339-2

17. Lin W, Zhao L, Huang X, et al. Comparative assessment of distribution characteristics and ocular pharmacokinetics of norvancomycin between continuous topical ocular instillation and hourly administration of eye drop. Drug Des Devel Ther. 2020;14:867-879. doi:10.2147/DDDT.S233047

18. Li J, He S, Yang Z, et al. Pharmacokinetics and cerebrospinal fluid penetration of norvancomycin in Chinese adult patients. Int $J$ Antimicrob Agents. 2017;49:603-608. doi:10.1016/j. ijantimicag.2017.01.014

19. Wu Y, Kang J, Wang Q. Drug concentrations in the serum and cerebrospinal fluid of patients treated with norvancomycin after craniotomy. Eur J Clin Microbiol Infect Dis. 2017;36:305-311. doi:10.1007/s10096-016-2803-9

20. Jiang Z, Lei X, Chen M, et al. Three structurally-related impurities in norvancomycin drug substance. J Antibiot (Tokyo). 2017;70:158-165. doi:10.1038/ja.2016.115

21. Duan YQ, Yang YZ, Huang XT, et al. Research on the comparison of the demethylvancomycin's diffusion-deposition characteristics in the ocular solid tissues of sustained subtenon drug delivery with subconjunctival injection. Drug Deliv. 2017;24:92-98. doi:10.1080/ 10717544.2016.1230904

22. Bachu R, Chowdhury P, Al-Saedi Z, et al. Ocular drug delivery barriers - role of nanocarriers in the treatment of anterior segment ocular diseases. Pharmaceutics. 2018;10(1):28. doi:10.3390/ pharmaceutics 10010028

23. Janagam DR, Wu L, Lowe TL. Nanoparticles for drug delivery to the anterior segment of the eye. Adv Drug Deliv Rev. 2017;122:31-64. doi:10.1016/j.addr.2017.04.001

24. Yellepeddi VK, Palakurthi S. Recent Advances in Topical Ocular Drug Delivery. J Ocul Pharmacol Ther. 2016;32:67-82. doi:10.1089/jop.2015.0047

25. Yousry C, Elkheshen SA, El-laithy HM, et al. Studying the influence of formulation and process variables on Vancomycin-loaded polymeric nanoparticles as potential carrier for enhanced ophthalmic delivery. Eur J Pharm Sci. 2017;100:142-154. doi:10.1016/j. ejps.2017.01.013

26. Bertens CJF, Gijs M, van den Biggelaar FJHM, et al. Topical drug delivery devices: a review. Exp Eye Res. 2018;168:149-160. doi:10.1016/j.exer.2018.01.010

27. Agarwal R, Iezhitsa I, Agarwal P, et al. Liposomes in topical ophthalmic drug delivery: an update. Drug Deliv. 2016;23:1075-1091. doi:10.3109/10717544.2014.943336

28. Wei S, Zhang C, Zhang S, et al. Treatment results of corneal collagen cross-linking combined with riboflavin and $440 \mathrm{~nm}$ blue light for bacterial corneal ulcer in rabbits. Curr Eye Res. 2017;42:1401-1406. doi:10.1080/02713683.2017.1332767

29. Kalra P, Ahirwar LK, Mittal R, et al. Clinical and histopathological evaluation of a rabbit model for pythium insidiosum keratitis. Curr Eye Res. 2020:1-8. doi:10.1080/02713683.2019.1676911 
30. Zaidi T, Reidy T, D’Ortona S, et al. CD74 deficiency ameliorates Pseudomonas aeruginosa-induced ocular infection. Sci Rep. 2011;1:1-7. doi:10.1038/srep00058

31. Shi Y, Lv H, Fu Y, et al. Preparation and characterization of a hydrogel carrier to deliver gatifloxacin and its application as a therapeutic contact lens for bacterial keratitis therapy. Biomed Mater. 2013:8. doi:10.1088/ $1748-6041 / 8 / 5 / 055007$.

32. Tas T, Kucukbayrak A, Hakyemez IN, et al. Linezolid versus vancomycin for the treatment of methicillin-resistant staphylococcus aureus keratitis in rabbits. Cornea. 2013;32:1052-1057. doi:10.1097/ ICO.0b013e3182912ffa

33. Bharti SK, Kesavan K. Phase-transition W/O Microemulsions for Ocular Delivery: evaluation of Antibacterial Activity in the Treatment of Bacterial Keratitis. Ocul Immunol Inflamm. 2017;25:463-474. doi:10.3109/09273948.2016.1139136

34. Wang M, Smith WA, Duncan JK, et al. Treatment of Pseudomonas keratitis by continuous infusion of topical antibiotics with the Morgan Lens. Cornea. 2017;36:617-620. doi:10.1097/ ICO.0000000000001128
35. Kesavan K, Kant S, Pandit JK. Therapeutic effectiveness in the treatment of experimental bacterial keratitis with ion-activated mucoadhesive Hydrogel. Ocul Immunol Inflamm. 2016;24:489-492. doi:10.3109/09273948.2015.1005238

36. Alvarez-Lorenzo C, Anguiano-Igea S, Varela-García A, et al. Bioinspired hydrogels for drug-eluting contact lenses. Acta Biomater. 2019;84:49-62. doi:10.1016/j.actbio.2018.11.020

37. Pinna A, Donadu MG, Usai D, et al. In vitro antimicrobial activity of a new ophthalmic solution containing povidone-iodine $0.6 \%$ $\left(\right.$ IODIM $^{\circledR}$ ). Acta Ophthalmol. 2020;98:e178-e180. doi:10.1111/ aos. 14243

38. Pinna A, Donadu MG, Usai D, et al. In vitro antimicrobial activity of a new ophthalmic solution containing hexamidine diisethionate 0.05\% (keratosept). Cornea. 2020;39:1415-1418. doi:10.1097/ ICO.0000000000002375

39. Chouhan S, Sharma K, Guleria S. Antimicrobial activity of some essential oils-present status and future perspectives. Medicine. 2017;4:58. doi:10.3390/medicines4030058

\section{Publish your work in this journal}

Drug Design, Development and Therapy is an international, peerreviewed open-access journal that spans the spectrum of drug design and development through to clinical applications. Clinical outcomes, patient safety, and programs for the development and effective, safe, and sustained use of medicines are a feature of the journal, which has also been accepted for indexing on PubMed Central. The manuscript management system is completely online and includes a very quick and fair peer-review system, which is all easy to use. Visit http://www. dovepress.com/testimonials.php to read real quotes from published authors. 